\title{
Quantifying the leading role of the surface state in the Kondo effect of $\mathrm{Co} / \mathrm{Ag}(111)$
}

\author{
M. Moro-Lagares,${ }^{1,2,3}$ J. Fernández,${ }^{4}$ P. Roura-Bas, ${ }^{4}$ M.R. Ibarra,,${ }^{1,5}$ A. A. Aligia,, , and D. Serrate ${ }^{1,5,0}$ \\ ${ }^{1}$ Instituto de Nanociencia de Aragón, Laboratorio de Microscopias Avanzadas, \\ University of Zaraqoza, E-50018 Zaragoza, Spain \\ ${ }^{2}$ Institute of Physics, Academy of Sciences, Prague, Czech Republic \\ ${ }^{3}$ Regional Centre of Advanced Technologies and Materials, Faculty of Science, \\ Department of Physical Chemistry, Palacky University, Olomouc, Czech Republic \\ ${ }^{4}$ Centro Atómico Bariloche and Instituto Balseiro, \\ Comisión Nacional deEnergía Atómica, 8400 Bariloche, Argentina \\ ${ }^{5}$ Departamento Física Materia Condensada, University of Zaraqoza, E-50018 Zaragoza, Spain
}

(Dated: July 6, 2018)

\begin{abstract}
Using a combination of scanning tunneling spectroscopy and atomic lateral manipulation, we obtained a systematic variation of the Kondo temperature $\left(T_{\mathrm{K}}\right)$ of Co atoms on $\mathrm{Ag}(111)$ as a function of the surface state contribution to the total density of states at the atom adsorption site $\left(\rho_{s}\right)$. By sampling the $T_{\mathrm{K}}$ of a Co atom on positions where $\rho_{s}$ was spatially resolved beforehand, we obtain a nearly linear relationship between both magnitudes. We interpret the data on the basis of an Anderson model including orbital and spin degrees of freedom (SU(4)) in good agreement with the experimental findings. The fact that the onset of the surface band is near the Fermi level is crucial to lead to the observed linear behavior. In the light of this model, the quantitative analysis of the experimental data evidences that at least a quarter of the coupling of Co impurities with extended states takes place through the hybridization to surface states. This result is of fundamental relevance in the understanding of Kondo screening of magnetic impurities on noble metal surfaces, where bulk and surface electronic states coexist.
\end{abstract}

PACS numbers: 72.15.Qm,73.22.-f,75.20.Hr,75.75.-c

\section{INTRODUCTION}

Single atoms with partially filled $d$ - or $f$-shells on a solid state surface are known to exhibit strong electron correlations leading to a wide range of physical ground states. The magnetic properties of such impurities on metals are inherently connected with many-body interactions between the localized magnetic moment and the conduction electrons $1-\underline{\underline{9}}$. In this framework, the Kondo effect $\frac{1,10,11}{1}$ is the most frequently found. Since this phenomenon is an archetypal example of the formation of a many-body quantum state, it is central in the understanding of the electronic behavior of complex strongly correlated electrons systems such as heavy fermions 11,12 , Kondo insulators 13 , and nanoscale systems $2,3,5,14-27$.

Thanks to the large spatial and energy resolution of scanning tunneling microscopy (STM) and spectroscopy (STS) ${ }^{2,18,19}$, these tools are extremely well suited to access the spectroscopic features of adsorbate induced many-body resonances in tunneling differential conductance $(d I / d V)$. Most of STM studies on Kondo impurities are performed on noble metal (111) surfaces, where both bulk and surface electrons coexist $2,3,5,19-28$. Unavoidably, the question of whether surface or the bulk electrons play the leading role in the Kondo effect raises. To date, the answer remains unclear because there are conflicting conclusions depending on the technical approach to the problem. Since bulk electrons decay much faster than surface state electrons into the crystal, it has been common practice to measure the Kondo resonance as a function of the lateral distance to the atom ${ }^{3,5,29,30}$.

For instance, Henzl et al $l_{\frac{5}{5}}$ concluded that bulk electrons determine the Kondo temperature $\left(T_{\mathrm{K}}\right)$ of $\mathrm{Co} / \mathrm{Ag}(111)$ by intentionally depleting the spectral weight of the surface state at Fermi level. The study of the Kondo resonance next to a monoatomic step edge led to the conclusion that the role of the surface states is marginal ${ }^{21}$. This is supported by the weak dependence of $T_{\mathrm{K}}$ of Co on noble metal surfaces ${ }^{31}$ with marked differences in the weight of their surface states relative to the bulk ones. On the contrary, the theoretically predicted 29,30 oscillations of the resonance line shape as a function of the tip lateral displacement on the order of the bulk electrons Fermi wavelength have not been observed $2.3 .5^{2}$. In fact, the theoretical description by Merino et $a l .32$ cannot explain the distance dependent data on $\mathrm{Co} / \mathrm{Cu}(111)^{\underline{3}}$ without a major involvement of the surface states.

The seminal work about the quantum mirage of the Kondo resonance into the focus of elliptical resonators proves unambiguously a finite contribution of surfaces states $^{20}$. Based on the relative intensity of $d I / d V$ at both foci (one with a Co impurity and the other empty) a lower bound of $1 / 10$ for the relative contribution of surface states has been estimated ${ }^{33}$. Moreover, the rather high $T_{\mathrm{K}} \sim 180 \mathrm{~K}$ of a Co porphirine on $(\sqrt{3} \times \sqrt{3}) \mathrm{Ag}$ $\mathrm{Si}(111)$, where bulk electrons states are not present, indicates that a significant coupling between the surface states and magnetic impurity is possible ${ }^{34}$. In support of this, it has been recently shown that $d I / d V$ of $\mathrm{Ag}(111)$ oscillates as the resonance width of Co atoms near step edges, quantum resonators or another atom ${ }^{35}$. It is worth noting that, from the theoretical point of view, the Kondo effect is extremely sensitive to the hybridiza- 
tion channels between the impurity and the metal host electrons, which exhibit non-trivial dependencies on the $\mathrm{k}$-space electronic structure of the surface and the actual adsorption geometry ${ }^{36}$. Thus, direct comparison of the Kondo resonance among different environments of the same adatom is physically inaccurate.

In this Article, we quantify the role of surface electron states in the Kondo effect of Co adatoms on $\mathrm{Ag}(111)$. We characterize their Kondo spectral features while varying just one single parameter of the problem: the surface state contribution to the local density of states of the substrate, $\rho_{s}$. In sections [II and III we develop the theoretical background on the basis of an Anderson model with $\mathrm{SU}(4)$ symmetry, which is consistent with the experimental spectroscopy as opposed to the $\mathrm{SU}(2)$ one $\mathrm{e}^{35}$. Section IV is devoted to the experimental differential conductance $d I / d V$ at position $\mathbf{R}$ with $\left(G_{K}\right)$ and without $(G)$ Co impurity between the tip and the $\operatorname{Ag}(111)$ surface. The analysis of $T_{\mathrm{K}}(\mathbf{R})$ and the amplitude of the Kondo resonance reveals that both magnitudes increase monotonically with $G(\mathbf{R})$. The theoretical calculation of the energy resolved $G$ for varying $\rho_{s}$ is given in section V] using both the non-crossing approximation (NCA) and poor man's scaling (PMS). Finally, in section VI the experimental and theoretical physical parameters are compared. We show that the coupling of the Co impurity state with extended states steaming from the surface state could be the dominant one, and prove a threshold of at least one fourth of that from the bulk states.

\section{SYMMETRY ANALYSIS}

In analogy with other nobel metal surfaces, $\frac{37}{,}$ the Co atoms might occupy two non equivalent hollow positions on the $\mathrm{Ag}(111)$ surface, depending on whether the Co atoms lie above a $\mathrm{Ag}$ atom of the second layer or not (fcc/hcp). In both cases the symmetry point group is $C_{3 v}$. This group has three irreducible representations: $A_{1}$ and $A_{2}$ of dimension one, and the two dimensional representation $E$. Disregarding spin for the moment, the Co $3 d$ orbitals are split in one $A_{1}$ singlet and two $E$ doublets, as sketched at the left side of Fig. 1, Choosing the coordinates in such a way that $z$ is perpendicular to the surface and one of the Ag atoms nearest to Co lies in the $x z$ plane, the $3 d$ orbital with symmetry $3 z^{2}-r^{2}$ transforms as the $A_{1}$ representation, $x z$ and $y z$ transform like the $E$ representation, and $x^{2}-y^{2}(-x y)$ transforms under the operations of $C_{3 v}$ in the same way as $x z(y z)$. Any Hamiltonian that respects the point group symmetry (and without additional symmetry) mixes these two doublets, leading to bonding and antibonding states. In particular, the antibonding $E$ states have the form

$$
\begin{aligned}
& \left|e_{1}\right\rangle=\alpha|x z\rangle+\beta\left|\left(x^{2}-y^{2}\right) / 2\right\rangle \\
& \left|e_{2}\right\rangle=\alpha|y z\rangle-\beta|x y\rangle .
\end{aligned}
$$

Additional adatoms on the surface break the $C_{3 v}$ symmetry, but this effect is small if these atoms are sufficiently far from the Co atom under study as is the case in this work.
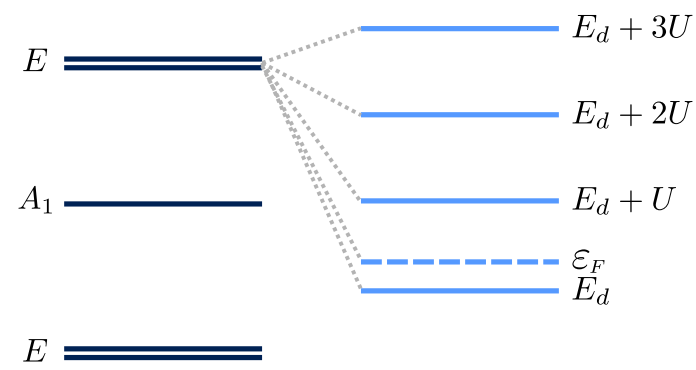

FIG. 1: Left: scheme of the splitting of the (one-particle) $3 d$ orbitals under the point group $C_{3 v}$. Right: scheme of the splitting of the four antibonding states of symmetry $E$ by the Coulomb repulsion. $\varepsilon_{F}$ denotes the position of the Fermi energy compatible with the position of the observed Fano antiresonance.

The Coulomb repulsion inside the $3 d$ orbitals splits the energy necessary to add electrons in the same orbital. For example, let us call $E_{d}$ the energy necessary to add the first electron in one of the antibonding $E$ orbitals with any spin. This energy does not depend on the particular antibonding orbital chosen $\left(e_{1}\right.$ or $\left.e_{2}\right)$ or its spin. However to add the second electron, one has to pay the Coulomb repulsion $U$ between them. Similarly, the necessary energy to add the third or fourth electron is $E_{d}$ plus the Coulomb repulsion with the previous ones. This is presented schematically at the right of Fig. 1] The actual position of the levels is more complex because it is modified by exchange and pair hopping terms (see for example Ref. 38), but they not affect our treatment. For example, for the ground state for occupancy 2 in the antibonding $E$ is a triplet due to Hund's rules. Instead, for occupancy 3 of theses states the ground state is degenerate and is formed by two spin doublets with one hole in either $e_{1}$ or $e_{2}$. A similar splitting takes place for the bonding $E$ and the $A_{1}$ states, which remain occupied in the neutral Co atom.

While symmetry alone cannot determine the ordering of the levels, the position of the observed Fano-Kondo dip $\omega_{\mathrm{K}}$ for positive energies of the order of the Kondo temperature $T_{\mathrm{K}}$ or larger (see for instance Figs. 20 and 5$)^{39}$ points to an $\mathrm{SU}(4)$ Kondo system with occupancy near 1 , as we show below. This in consistent with the configuration $3 d^{7}$ expected for a neutral Co atom, with four electron occupying the bonding $E$ orbitals, two in the $A_{1}$ orbital and the remaining electron in one antibonding $E$ orbitals (Fig. 1). Other possibilities can be disregarded. For example if both $A_{1}$ states were the highest in energy putting two holes there and one in the antibonding $E$ orbitals, the model presented in Section III still holds after an electron-hole transformation in the antibonding $E$ orbitals, in which case the Kondo dip would be to the left of the Fermi energ (i.e., same differential conductance 
as in Fig. 5 but with opposite sign for $\omega$ ). Assuming a $3 d^{8}$ configuration, one has two possibilities to obtain a Kondo state: i) two holes in the antibonding $E$ states, but in this case the Kondo dip would be centered at the Fermi level ${ }^{40}$, ii) one hole in an $E$ state and one hole in an $A_{1}$ state. This is the case of Fe phtalocyanine on $\mathrm{Au}(111)$ which shows a two-stage Kondo effect with two

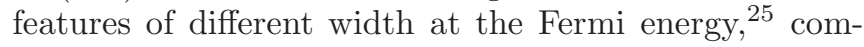
pletely different from our case. We have not discussed above combinations of holes in bonding and antibonding $E$ orbitals because they are unlikely for Co.

Therefore two channels are necessary to describe the system and one-channel models [like the ordinary onechannel SU(2) Anderson or Kondo model] are ruled out. One has in principle a spin $\mathrm{SU}(2)$ times orbital $\mathrm{SU}(2)$ model. However, for large $U$ (we take $U \rightarrow \infty$ but this is not an essential approximation ${ }^{41}$ ) the symmetry is $\mathrm{SU}(4)$ (larger than $\mathrm{SU}(2) \times \mathrm{SU}(2)$ ), including orbital and spin degeneracies.

\section{MODEL AND FORMALISM}

\section{A. Hamiltonian}

The Hamiltonian can be written as

$$
\begin{aligned}
H= & \sum_{k i \sigma} \varepsilon_{k}^{s} s_{k i \sigma}^{\dagger} s_{k i \sigma}+\sum_{k \sigma} \varepsilon_{k}^{b} b_{k i \sigma}^{\dagger} b_{k i \sigma}+E_{d} \sum_{\sigma} d_{i \sigma}^{\dagger} d_{i \sigma}+ \\
& +U \sum_{i \sigma \neq j \sigma^{\prime}} d_{i \sigma}^{\dagger} d_{i \sigma} d_{j \sigma^{\prime}}^{\dagger} d_{j \sigma^{\prime}}+\sum_{k \sigma} V_{k}^{s}\left[d_{i \sigma}^{\dagger} s_{k i \sigma}+\text { H.c. }\right]+ \\
& +\sum_{k \sigma} V_{k}^{b}\left[d_{i \sigma}^{\dagger} b_{k i \sigma}+\text { H.c. }\right] .
\end{aligned}
$$

where $d_{i \sigma}^{\dagger}$ creates an electron in the antibonding orbital $\left|e_{i}\right\rangle$ with spin $\sigma$, and $s_{k i \sigma}^{\dagger}\left(b_{k i \sigma}^{\dagger}\right)$ are creation operators for an electron in the $k^{t h}$ surface (bulk) conduction eigenstate with symmetry $i$ and spin $\sigma$.

We assume constant densities of bulk states $\rho_{b}$ extending in a wide range from $-D$ to $D$, and $\rho_{s}$ extending from $D_{s}$ to $D\left(\left|D_{s}\right|<D\right)$. As we shall show, the fact that the surface band begins abruptly near the Fermi level at $D_{s}=-67 \mathrm{meV}^{42}$ (neglected in alternative treatments ${ }^{35}$ ) plays an essential role in the interpretation of the results. We also assume constant hybridizations $V_{b}=V_{k}^{b}$ and $V_{s}=V_{k}^{s}$. We believe that these assumptions are not crucial as long as the dependence of these parameters on energy is smooth in a range of a few times $T_{\mathrm{K}}$ around the Fermi energy. We define the couplings of the impurity state to bulk and surface state electrons as $\Delta_{b}=\pi \rho_{b}\left|V_{k}^{b}\right|^{2}$ and $\Delta_{s}=\pi \rho_{s}\left|V_{k}^{s}\right|^{2}$ respectively. Our work allows to experimentally determine the ratio of these two quantities. $E_{d}$ is the energy of the relevant impurity state.

We solve the model using two techniques: non-crossing approximation (NCA, section $\overline{\mathrm{VA}}{ }^{11,43}$ and poor man's scaling (PMS, section $\overline{V B})^{11}, \underline{44}$ on the effective CoqblinSchrieffer model. These approaches are known to reproduce correctly the relevant energy scale $T_{\mathrm{K}}$ and its dependence on the Anderson parameters. In contrast to Numerical Renormalization Group in which the logarithmic discretization of the conduction band 45,46 broadens finite-energy features $\underline{46.47}$, and leads to inaccurate Kondo temperatures when a step in the conduction band is near the Fermi level, NCA correctly describes these features. For instance, the intensity and the width of the charge-transfer peak of the spectral density (the one near $E_{d}$ ) was found $\frac{48,49}{}$ in agreement with other theoretical methods $\underline{\underline{4}-51}$ and experiment $\underline{52}$. The NCA works satisfactorily in cases in which the density of conduction states is not smooth $\frac{53}{53}$, including in particular a step in the conduction band $\stackrel{54}{ }$. Furthermore, it has a natural extension to non-equilibrium conditions $s^{\frac{55}{3}}$ and it is specially suitable for describing satellite peaks of the Kondo resonance, as those observed in Ce systems ${ }^{56.57}$, or away from zero bias voltage in non-equilibrium transport $\underline{58-61}$. Due to shortcomings of the approximation for finite $U \underline{\underline{50,62,63}}$, we restrict our calculations to $U \rightarrow \infty$ but this is not an essential approximation in our case $\underline{41}$.

The PMS is a perturbative approach that integrates out progressively a small portion of the conduction states lying at the bottom and at the top of the conduction bands, renormalizing the Kondo exchange coupling $J_{K} \underline{11.44}$.

\section{B. The STM tunneling conductance}

The differential conductance $d I / d V$ is proportional to the spectral density of the mixed state $h_{i \sigma}\left(\mathbf{R}_{t}\right)$ at the position of the STM tip $\mathbf{R}_{t}$.

$$
\begin{aligned}
G_{K}(e V) & =d I / d V \propto \sum_{i \sigma} \rho_{h i \sigma}(e V), \\
\rho_{h i \sigma}(\omega) & =\frac{1}{2 \pi j}\left[\tilde{G}_{h i \sigma}(\omega-j \epsilon)-\tilde{G}_{h i \sigma}(\omega+j \epsilon)\right], \\
h_{i \sigma}\left(\mathbf{R}_{t}\right) & =\frac{1}{N}\left[s_{i \sigma}\left(\mathbf{R}_{t}\right)+p_{b} b_{i \sigma}\left(\mathbf{R}_{t}\right)+p_{d} d_{i \sigma}\left(\mathbf{R}_{t}\right)\right] .
\end{aligned}
$$

where $V$ is the sample bias potential of the STM, $e$ the electron elementary charge, $\tilde{G}_{h i \sigma}(\omega)=\left\langle\left\langle h_{i \sigma} ; h_{i \sigma}^{\dagger}\right\rangle\right\rangle_{\omega}$ is the Green's function of $h_{i \sigma}\left(\mathbf{R}_{t}\right), j$ is the imaginary unit, $\epsilon$ is a positive infinitesimal, $N$ is a normalization factor, $p_{b}$ is the ratio of the tunneling matrix element between the STM tip and the bulk states $b_{i \sigma}$ and between tip and surface states $s_{i \sigma}$, while $p_{d}$ is the analogous ratio for Co state $d_{i \sigma}\left(\mathbf{R}_{t}\right)$ and surface states at the tip position. $h_{i \sigma}\left(\mathbf{R}_{t}\right)$ represents the linear combination of surface, bulk and Co $3 \mathrm{~d}$ states probed by the tip.

Using equations of motion, $\rho_{h i \sigma}(\omega)$ can be related with the Green's function for the $d$ electrons $\tilde{G}_{d i \sigma}(\omega)=$ $\left\langle\left\langle d_{i \sigma} ; d_{i \sigma}^{\dagger}\right\rangle\right\rangle_{\omega}$, and the unperturbed Green's functions for conduction/bulk electrons $\tilde{G}_{s / b}^{0}(\omega)$. In absence of mag- 
netic and symmetry-breaking fields we can drop the subscripts $i \sigma$ :

$$
\tilde{G}_{h}(\omega)=\tilde{G}_{s}^{0}(\omega)+p_{b}^{2} \tilde{G}_{b}^{0}(\omega)+\Delta \tilde{G}_{h}(\omega)
$$

$\Delta \tilde{G}_{h}(\omega)=0$ if the Co impurity is absent and if not

$$
\begin{aligned}
\Delta \tilde{G}_{h}(\omega) & =F^{2}(\omega) \tilde{G}_{d}(\omega), \\
F(\omega) & =V_{s} \tilde{G}_{s}^{0}(\omega)+p_{b} V_{b} \tilde{G}_{b}^{0}(\omega)+p_{d},
\end{aligned}
$$

where

$$
\begin{aligned}
& \tilde{G}_{b}^{0}\left(R_{i}, R_{i}, \omega\right)=\rho_{b}\left[\ln \left(\frac{\omega+D}{\omega-D,}\right)\right], \\
& \tilde{G}_{s}^{0}\left(R_{i}, R_{i}, \omega\right)=\rho_{s}\left[\ln \left(\frac{\omega-D_{s}}{\omega-D}\right)\right] .
\end{aligned}
$$

\section{EXPERIMENTAL RESULTS}

Single Co atoms were deposited at low temperatures onto the $\mathrm{Ag}(111)$ surface $\left(T_{e v} \sim 3 \mathrm{~K}\right.$ for an experimental temperature $T=1.1 \mathrm{~K}$ ) cleaned by repeated cycles of sputtering with $\mathrm{Ar}^{+}$and annealing at $500{ }^{\circ} \mathrm{C}$ in $\mathrm{UHV}$ $\left(P_{\text {base }} \leq 1 \times 10^{-10}\right.$ mbar $)$. We use a lock in amplifier to perform STS as a function of the applied sample bias, $V$. STS was acquired at constant height defined by the regulation set point $V_{0}, I_{0}$ on $\operatorname{Ag}(111)$ with rms modulation voltage $V_{\text {mod }}$ and implemented in two modes: (i) Single point $d I / d V$ spectroscopy $\left(V_{\bmod }=0.5 \mathrm{mV}, V_{0}=-100\right.$ $\mathrm{mV}, I_{0}=42 \mathrm{pA}$ ) on top of Co atoms to obtain the energy resolved $G_{K}(\mathbf{R})$; and (ii) $d I / d V(x, y)$ mapping at Fermi level $\left(V_{\text {mod }}=1 \mathrm{mV}, V_{0}=-100 \mathrm{mV}, I_{0}=200 \mathrm{pA}\right)$ to measure the spatially resolved $G(\mathbf{R})$ of the $\operatorname{Ag}(111)$ inspected area after clearing it away from atoms by means of atomic manipulation (typical set point for manipulation $V_{0}=3 \mathrm{mV}, I_{0}=40-70 \mathrm{nA}$ ). The working temperature is $T=1.1 \mathrm{~K}$ or $T=4.7 \mathrm{~K}$, being the Kondo features of one atom in STS identical at both temperatures.

Experimentally, the Kondo effect of isolated Co atoms on metals manifests as a Fano resonance, $2,19,31,64$ in the impurity $G_{K}$ near the Fermi level. We describe this singularity as $G_{K}=\mathcal{G}_{0} g_{K}$, where $\mathcal{G}_{0}(\mathbf{R}, \omega)$ is the convolution of the tip and the impurity density of states in absence of Kondo screening and $g_{K}(\mathbf{R}, \omega)$ contains the Fano function, $\mathcal{F}(x, q)=(x+q)^{2} / 1+x^{2}$, as follows:

$$
g_{K}(\mathbf{R}, \omega)=\left(1-A_{K}(\mathbf{R})\right)+A_{K}(\mathbf{R}) \mathcal{F}\left[\frac{\omega-\omega_{K}}{\Gamma_{0}(\mathbf{R}) / 2}, q\right]
$$

Here $\omega=e V, \omega_{K}$ the energy of the center of the Kondo resonance, $q$ the Fano asymmetry factor, $A_{K}(\mathbf{R})$ the resonance amplitude when the atom sits at surface position $\mathbf{R}$, and $\Gamma_{0}(\mathbf{R})$ the resonance width, which is related to the Kondo temperature as $2 k_{B} T_{\mathrm{K}} \simeq \Gamma_{0}$ for $T / T_{\mathrm{K}} \rightarrow 0^{19,65}$. Below $T_{\mathrm{K}}$ the spin of the extended
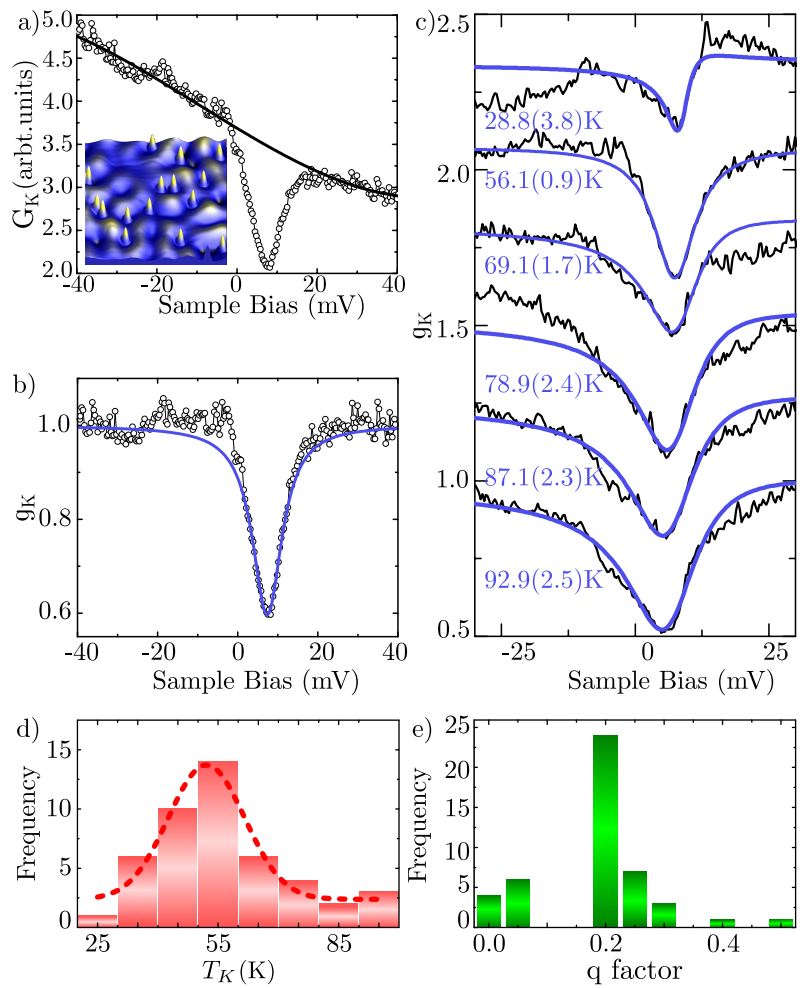

FIG. 2: (a) Representative raw $\mathrm{d} I / \mathrm{d} V\left(G_{K}(V)\right.$, empty circles) showing the Kondo zero bias feature at the center of a single Co atom and background estimation $\left(\mathcal{G}_{0}(V)\right.$, solid line). (b) Fit of the resulting $g_{K}(V)$ to equation 7 yielding $T_{\mathrm{K}}=56.1 \pm 0.9 \mathrm{~K}, q=0$ and $\omega_{K}=7.39 \pm 0.04 \mathrm{meV}$. (c) Dispersion found in the Kondo resonance of set of atoms spread on $\operatorname{Ag}(111)$ with the corresponding fit and $T_{\mathrm{K}}$ value. (d-e) Kondo Temperature and $q$ factor statistics. Using a Gaussian distribution profile (dashed line) for the $T_{\mathrm{K}}$ histogram, we obtain $\left\langle T_{K}\right\rangle=52.1 \pm 9.4 \mathrm{~K}$.

states couples antiferromagnetically and screens the impurity spin, giving rise to the Kondo state 10,11 . Figures 2(a-b) shows the analysis of a Kondo resonance based on Eq. (7), which permits to extract the parameters $T_{\mathrm{K}}(\mathbf{R})$, $A_{K}(\mathbf{R}), q$ and $\omega_{K}$ for each individual atom at position R.

We first analyze $G_{K}$ of several Co atoms dispersed over the surface at their position right after the evaporation process (i.e., prior to any atom repositioning with the tip). Figures 2(c-e) unveil a significant uncertainty in the parameters describing the Kondo resonance. The histograms elaborated from a set of 40 different atoms are shown in Figures 2(d-e). $T_{K}$ spans over a range of $28 \mathrm{~K} \leq T_{\mathrm{K}} \leq 95 \mathrm{~K}$, with $\left\langle T_{\mathrm{K}}\right\rangle=52.1 \pm 9.4 \mathrm{~K}$ being the most probable value. The most frequently found value for $A_{K}$ and $q$ is 0.2 .

Apart from the $h c p / f c c$ character of the hollow sites in a (111) surface termination, the adsorption geometry of disperse Co atoms is indistinguishable. We have confirmed that the Kondo parameters are the same in both sites except for a slightly lower amplitude $A_{K}$ in 


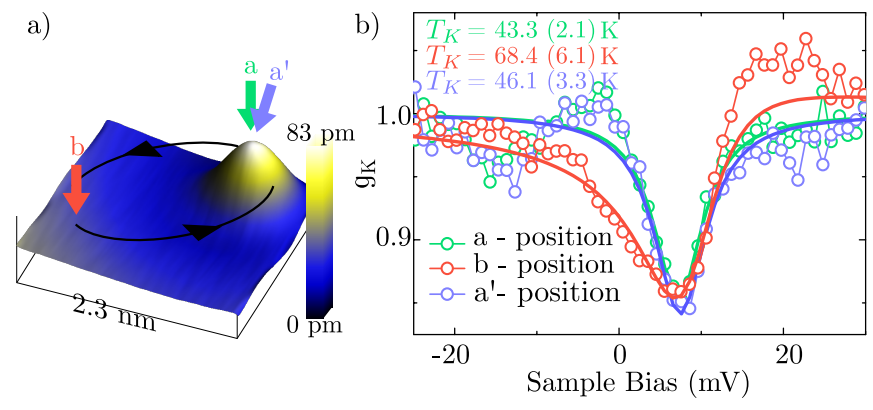

FIG. 3: a) Representation of the experiment carried out in the study of the variations of the Kondo resonance with the point contact $\rho_{s}$. b) $\mathrm{d} I / \mathrm{d} V$ spectrum (circles) and fit to Eq. (7) of the Co atom at $a$-position (green); $b$-position (red) and $a^{\prime}$-position (blue). $a^{\prime}$-position is the same as the $a$-position but after atomic manipulation.

one of them. Therefore, the different values obtained for $T_{K}$ and $q$ suggest a sensitivity to the density of surface states $\rho_{s}(\mathbf{R})$. Particularly, in $\operatorname{Ag}(111)$, the onset of surface state lies in close proximity $\left(D_{s}=-67 \mathrm{meV}\right)$ to the Fermi level ${ }^{42}$, leading to a Fermi wavelength $\lambda_{F} \sim 8$ $\mathrm{nm}^{28}$, which is comparable to the distance between surface scatterers such as step edges, point impurities or Co adatoms. This will produce interference patterns in $\rho_{s}(\mathbf{R})$ with a characteristic length scale of $\lambda_{F} / 2$. We have shown elsewhere ${ }^{28}$ that $\rho_{s}$ contributes strongly to the total density of states $h_{i \sigma}\left(\mathbf{R}_{t}\right)\left(\rho_{h}\right)$ probed by the tip. Therefore, it is natural to expect that changes in $\rho_{s}(\mathbf{R})$ lead to the observed dispersion of $T_{K}$ of $\mathrm{Co} / \mathrm{Ag}(111)$, through the hybridization of the Co $3 \mathrm{~d}$ electrons with the surface states. This will become clear in Section $\mathrm{VB}$ where an analytical expression for the dependence of $T_{K}$ with $\rho_{s}$ is presented.

To benchmark the correlation of $T_{K}$ with the electronic properties of the substrate, we measure $g_{K}$ (see Eq. (77) over a Co atom at its natural adsorption site $\mathbf{R}$, and subsequently at another position $\mathbf{R}^{\prime}$ far enough as to have presumably a different $\rho_{s}\left(\left|\mathbf{R}-\mathbf{R}^{\prime}\right| \sim \lambda_{F} / 2\right)$. In Fig. 3 we show $g_{K}$ at each site and in the absence of any tip change during the manipulation procedure. We find a strong variation of $\Delta T_{K}=T_{\mathrm{K}}\left(\mathbf{R}^{\prime}\right)-T_{\mathrm{K}}(\mathbf{R})=24 \pm 4$ $\mathrm{K}$, well above the experimental uncertainty. This experiment shows unambiguously that the coupling strength between the localized spin and the Fermi gas of conduction electrons is strongly influenced by the local value of $\rho_{s}$ at each contact point.

Next, we evaluate more precisely this position dependent Kondo effect through the analysis of $T_{\mathrm{K}}$ and $A_{K}$ of Co atoms relocated in a region where $G(\mathbf{R})$ at Fermi level has been previously characterized (without Co atoms) in constant height conditions. First, we clean out the atoms in the selected working area as depicted in Figs. $4(a, b)$. Second we take a $G(\mathbf{R})$ image of the differential conductance near Fermi level $(0<V<3 \mathrm{mV})$ as shown in Fig. 45, whose maxima and minima reflect the characteristic interference pattern of the surface state. After- wards, a single Co atom is moved across the inspected $\mathrm{Ag}(111)$ area (Fig. $4 \mathrm{~d})$ and we measure its energy spectrum $G_{K}(\mathbf{R})$ for each $\mathbf{R}$ location. Note that this procedure is free of feedback artifacts, and that the drift between consecutive images is corrected by referring always $\mathbf{R}$ to a reference feature of the same image.

At the tip-sample distance at which the experiment is performed the STM does not exhibit atomic resolution. Thus, $G(\mathbf{R})$ oscillations are only contributed by $\rho_{s}(\mathbf{R})$, owing to the interference pattern of scattered surface state quasiparticles. In Figs. 四(e,f) we plot $T_{K} /\left\langle T_{K}\right\rangle$ and $A_{K}$ as a function of $G / G_{0}$ for four different data sets gathered together, taken with different tips (symbol code) at different working areas (color code). $G_{0}$ is defined as the tunneling conductance of an ideal surface without scattering sources. Experimentally, we determine $G_{0}$ as the average differential conductance at Fermi level of a region much larger than $\lambda_{F}$, as the one shown in Fig. 45. This normalization makes the analysis insensitive to the specific electronic structure of the tips used for the experiment. The resulting graphs display a monotonic increase of $T_{\mathrm{K}}$ and $A_{K}$ with $G$, which implicitly provides an evidence of the linear dependence of these parameters on $\rho_{s}$ within the experimental boundaries.

\section{THEORETICAL RESULTS}

In this section we present the theoretical results for the dependence of $T_{\mathrm{K}}$ on the surface states density, $\rho_{s}$. For simplicity, from now on we choose the origin of energies at $\varepsilon_{F}=0$. We have taken $D_{s}=-67 \mathrm{meV}$ from experiment $21,28,66$ and have chosen $D=4 \mathrm{eV}, \rho_{b}=0.135$ $\mathrm{eV}^{-1}, \rho_{s}=0.0446 \mathrm{eV}^{-1}$ (Ref. 28). The results are rather insensitive to these parameters if the hybridizations are changed to fix the values of $\Delta_{b}$ and $\Delta_{s}$. For the energy of the occupied antibonding $E$ state with majority spin (see Fig. (1), we take $\left|E_{d}\right| \gg \Delta_{s, b}$ (in particular $E_{d}=-2.2$ ). A different value would simply require a rescaling of $\Delta_{s, b}$.

Concerning the parameters entering Eq. (3), previous comparison between experiment and theory on the action of Co resonators on the surface states 28 suggest that $p_{b}^{2} \approx 1 / 16$. At first we have taken $\left|p_{b}\right|=1 / 4$, but this implies a very large surface contribution $\left(\Delta_{s}^{0} / \Delta_{b}>6\right.$, see below). Furthermore, this estimation applies to a different tunneling barrier height ${ }^{28}$, which may strongly alter the ratio $p_{b}$. Therefore we think that it is better to be cautious and treat $p_{b}$ as an unknown parameter. The shape of the resulting differential conductance $d I / d V$ is rather insensitive to the sign of $p_{b}$ but the intensity is smaller for $p_{b}<0$. The parameter $p_{d}$ is determined by fitting the line shape. The line shape is rather insensitive to $p_{b}$ if $p_{d}$ is adjusted. 

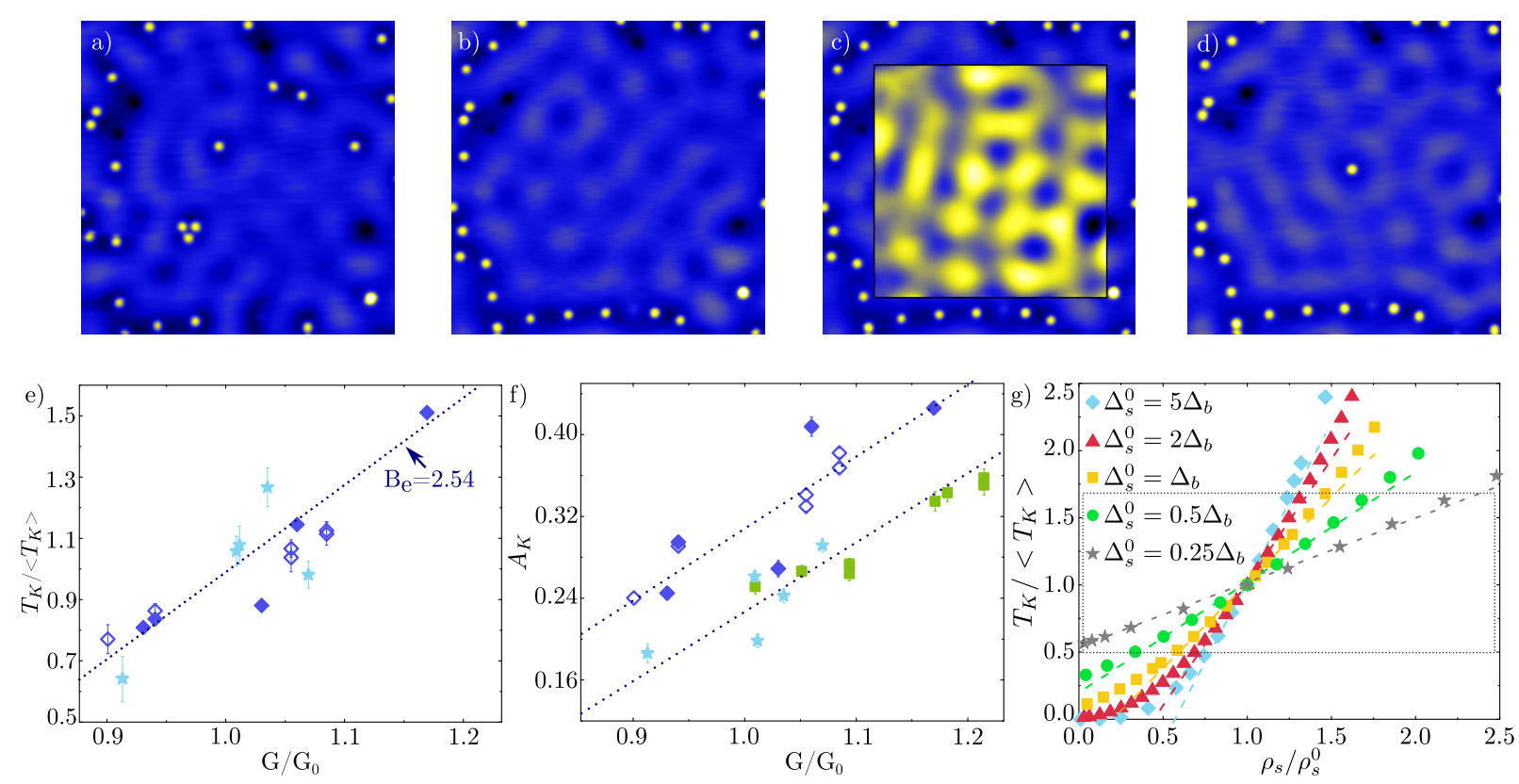

FIG. 4: $T_{K}$ and $A_{K}$ variations with $G(\mathbf{R})$. a) STM image of Co/Ag(111) after Co deposition. b) Co atoms are removed from the working area to avoid their influence on $G$. c) Inset: constant height $G(\mathbf{R})=d I / d V$ map taken at $V=3 \mathrm{mV}\left(I_{0}=200 \mathrm{pA}\right.$, $V_{0}=-100 \mathrm{mV}$, and $V_{\text {mod }}=1 \mathrm{mV}$ ). d) Co atom relocated at a certain position over the surface. e) Experimental dependence of $T_{K} /\left\langle T_{K}\right\rangle$ on the normalized local tunneling conductance, $G / G_{0}$. f) Experimental dependence of $A_{K}$ on $G / G_{0}$. In e) and f), the color-code represents data sets taken with the same tip, while for the same color, the opened/closed circle-code distinguishes data sets at two nearby different working areas. All measurements were taken in constant height mode at $T=1.1 \mathrm{~K}\left(I_{0}=42\right.$ $\mathrm{pA}, V_{0}=-100 \mathrm{mV}$, and $\left.V_{\text {mod }}=0.5 \mathrm{mV}\right)$. g) Theoretical dependence of $T_{K} /\left\langle T_{K}\right\rangle$ on $\rho_{s} / \rho_{s}^{0}$ for different values of $\Delta_{s}^{0} / \Delta_{b}$. The dotted lines are linear fits in the region $0.5<T_{K} /\left\langle T_{K}\right\rangle<1.5$. The region enclosed within the dotted rectangle corresponds to the experimental parameter range.

\section{A. Non-crossing approximation}

\section{Calculation of the Kondo temperature}

To determine theoretically the value of the Kondo temperature $T_{\mathrm{K}}$, we calculate the conductance through the magnetic impurity as a function of temperature $G_{d}(T)$ for a hypothetical case with $p_{d} \rightarrow \infty$ and look for the temperature such that $G_{d}\left(T_{K}\right)=\gamma_{0} / 2$, where $\gamma_{0}$ is the ideal conductance of the system (reached for $T=0$ and occupancy 1 of the impurity level). Alternative definitions of $T_{\mathrm{K}}$ differ in factor of the order of $1 \frac{67}{}$, which is not relevant to us, as we shall show. We are interested in the dependence of $T_{\mathrm{K}}$ with $\Delta_{s}$. In practice we take

$$
G_{d}(T)=\gamma_{0} \frac{\pi \Delta}{2} \int d \omega\left(-\frac{\partial f(\omega)}{\partial \omega}\right) \rho_{d}(\omega),
$$

where $\Delta=\Delta_{b}+\Delta_{s}, \rho_{d}(\omega)=\sum_{i \sigma} \rho_{d i \sigma}(\omega)$ is the total impurity spectral density adding both orbitals $i$ and spins $\sigma$, and $f(\omega)$ is the Fermi function.

\section{Fit of the experimental data}

In Fig. 5 we show one experimental result for the differential conductance for which the resulting $T_{\mathrm{K}}$ is very

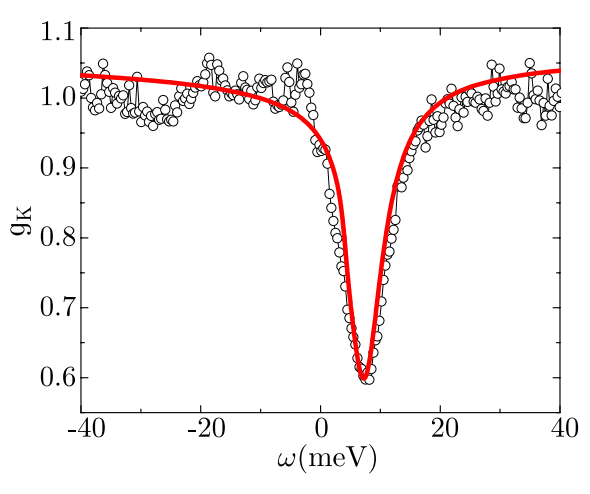

FIG. 5: (Color online) Differential conductance as a function of voltage. Open circle: experimental $g_{k}$ (same as Fig. 2b) without background. Red line: theory for $\Delta_{s}=69.26 \mathrm{meV}$, $\Delta_{b}=256.5 \mathrm{meV}, p_{b}=1.16$, and $p_{d}=7$.

near to the average one $\left\langle T_{\mathrm{K}}\right\rangle$, and the corresponding theoretical fit obtained at the experimental temperature $T=4.7 \mathrm{~K}$. For the latter, we have assumed $\Delta_{s}=0.27 \Delta_{b}$, $p_{b}=1.16$, which is consistent with the experimental slope of $T_{\mathrm{K}}$ vs. the tunneling conductance (see below) and adjusted $p_{d}$ to fit the experimental data. Very similar fits are obtained for larger values of $p_{b}$. The fit requires to shift the theoretical results by $4 \mathrm{meV}$ to reach the experimental position of the $\operatorname{dip} \omega_{K}=7.7 \mathrm{meV}$. The reason 
of this discrepancy might be due to details on the energy dependence of $\Delta_{s}$, which are particularly sensitive to the position of the adatoms ${ }^{28}$ and we have neglected in our approach.

Note that for the parameters in Fig. 5, the total width of the Fano dip is $\Gamma_{0}=8.71 \mathrm{meV}$, while twice $T_{K}$ obtained from the definition based on Eq. (8) gives $2 k_{\mathrm{B}} T_{\mathrm{K}}=9.78 \mathrm{meV}$. This ratio is approximately constant for the different parameters used here. Our Fano fit for this experimental curve gives $T_{K}=56.1 \mathrm{~K} \sim 4.83 \mathrm{meV}$. Therefore we assume that this value is representative of the average Kondo temperature $\left\langle T_{K}\right\rangle$ observed in experiment. Note that the ratio $T_{K} /\left\langle T_{K}\right\rangle$ does not depend on the definition of $T_{K}$. We define $\rho_{s}^{0}$ and $\Delta_{s}^{0}=\pi \rho_{s}^{0} V_{s}^{2}$ as the values of the surface spectral density and $\Delta_{s}$ that lead to $T_{\mathrm{K}}=\left\langle T_{\mathrm{K}}\right\rangle$. $T_{\mathrm{K}}$ depends mainly on $\Delta_{s}+\Delta_{b}$ and several ratios $\Delta_{s} / \Delta_{b}$ can lead to the same $T_{\mathrm{K}}$.

In Fig. $4 \mathrm{~s}$ we show the dependence of $T_{\mathrm{K}} /\left\langle T_{\mathrm{K}}\right\rangle$ vs. $\Delta_{s} / \Delta_{s}^{0}=\rho_{s} / \rho_{s}^{0}$ for several values of $R=\Delta_{s}^{0} / \Delta_{b}$. In good agreement with the experimental behavior of $T_{\mathrm{K}}$ (Fig. 45), we obtain a linear trend in the interval $0.5<$ $T_{\mathrm{K}} /\left\langle T_{\mathrm{K}}\right\rangle<1.5$ with slope $B$. As expected, $B$ increases with increasing $R=\Delta_{s}^{0} / \Delta_{b}$. For larger $R$ the linear dependence weakens and some curvature appears. The results for the slope $B$ for different ratios $R=\Delta_{s}^{0} / \Delta_{b}$ and what it implies for $p_{b}$ are listed in Table $\square$.

TABLE I: Slope of $T_{K} /\left\langle T_{K}\right\rangle$ vs. $\rho_{s} / \rho_{s}^{0}$, the corresponding $C_{b}$ value (see section $\mathrm{VI}$ ) and coefficient of the bulk density of states in Eq. (5) for different ratios $R=\Delta_{s}^{0} / \Delta_{b}$.

\begin{tabular}{cccc}
\hline$R$ & $B$ & $C_{b}$ & $p_{b}$ \\
\hline \hline 0.25 & 0.480 & 4.289 & 1.190 \\
0.27 & 0.503 & 4.045 & 1.156 \\
0.5 & 0.820 & 2.098 & 0.832 \\
1 & 1.269 & 1.001 & 0.575 \\
2 & 1.835 & 0.384 & 0.356 \\
5 & 2.375 & 0.070 & 0.152 \\
\hline
\end{tabular}

\section{B. Poor man's scaling}

The PMS11,44 for this SU(4) problem (or in general for $\mathrm{SU}(N)$ symmetry) up to second order in the CoqblinSchrieffer interaction $J_{K}$ has the same form as for the $\mathrm{SU}(2)$ Kondo Hamiltonian treated previously ${ }^{54}$, taking $N J_{K}$ as the interaction constant. Then, borrowing previous results and taking the limit $U \rightarrow \infty$ we obtain the following analytical formula for the Kondo temperature as a function of $\Delta_{b}$ and $\Delta_{s}$

$$
\begin{aligned}
T_{K} & \simeq A\left|D_{s}\right|^{\eta} D^{1-\eta} \exp \left[\frac{\pi E_{d}}{4\left(\Delta_{b}+\Delta_{s}\right)}\right], \\
\eta & =\frac{\Delta_{s}}{\left(\Delta_{b}+\Delta_{s}\right)} .
\end{aligned}
$$

where for second order in $J_{K}, A=1$. Higher order corrections reduce $A$ and introduce logarithmic corrections. However, in our case, it is not possible to obtain an analytical formula like Eq. (9) if these corrections are included.

In Fig. 6 we plot this function for the same parameters of Fig. 4s, showing again a linear dependence in the relevant range of parameters, in agreement with experiment. We obtain a semiquantitative agreement with the NCA (which assumes $U \rightarrow \infty$ ).

Eq. (9) sheds light on the expected dependence of $T_{\mathrm{K}}$ as a function of $\Delta_{s}$. In the experimentally relevant range of parameters the last (exponential) factor has a marked upward curvature which is largely compensated by the factor $\left|D_{s}\right|^{\eta} D^{1-\eta}$ leading to the approximately linear dependence displayed in Fig. 6. Replacing $\left|D_{s}\right|^{\eta} D^{1-\eta}$ by $D \sim 4 \mathrm{eV}$ (as in Ref. 35) cannot reproduce our experimental results.

In Table II we display the slope $(B)$ obtained from a linear fit in the interval $0.5<T_{K} /\left\langle T_{K}\right\rangle<1.5$. The slope with NCA is about $13 \%$ (for lower $R$ ) to $20 \%$ (for larger $R$ ) larger than with PMS (cf. Tables \and II). The agreement might be improved including numerically logarithmic corrections of order $J_{K}^{3}$ but we failed in the attempt to calculate them.

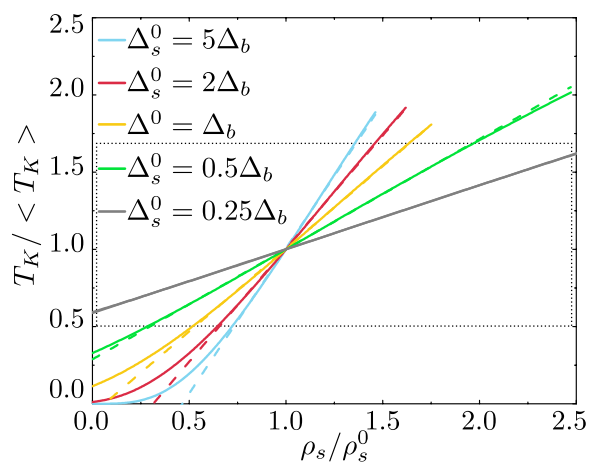

FIG. 6: (Color online) Same as Fig. 4s using Eq. (9). $T_{K}^{0}$ is the value obtained for the same parameters as before, and differs from the value of $\left\langle T_{\mathrm{K}}\right\rangle$ by a factor $\sim 2$. The region enclosed within the dotted rectangle corresponds to the experimental parameter range.

TABLE II: Same as Table I calculated with PMS.

\begin{tabular}{cccc}
\hline$R$ & $B$ & $C_{b}$ & $p_{b}$ \\
\hline \hline 0.25 & 0.414 & 5.135 & 1.302 \\
0.27 & 0.445 & 4.709 & 1.247 \\
0.5 & 0.713 & 2.562 & 0.920 \\
1 & 1.070 & 1.374 & 0.674 \\
2 & 1.465 & 0.734 & 0.492 \\
5 & 1.878 & 0.352 & 0.341 \\
\hline
\end{tabular}




\section{QUANTITATIVE DISCUSSION}

We have obtained experimentally and theoretically a linear trend of $T_{\mathrm{K}}\left(\rho_{s}\right)$. This might be surprising at first sight, since an exponential dependence of $T_{\mathrm{K}}$ with the coupling strength is expected 68,69 . However, due to the always existing bulk contribution, the proximity of the bottom of the surface band to the Fermi level and the particular region of interest of the parameter phase space (see the analytical PMS result in Eq. (9)), the expected upward curvature is strongly reduced, particularly for small $R$.

Since the measurements are performed at constant height, the experimental $d I / d V$ without a Co impurity can be written for all positions as $G=C\left(\rho_{s}+p_{b}^{2} \rho_{b}\right)$ (from Eqs. (3) and (41) with $\Delta \tilde{G}_{h}(\omega)=0$ ). Here $C$ and $\rho_{b}$ are constants. We write it in the form $G=$ $C \rho_{s}^{0}\left(\rho_{s} / \rho_{s}^{0}+C_{b}\right)$, where $C_{b}=p_{b}^{2} \rho_{b} / \rho_{s}^{0}$ is the relative weight of the bulk states in tunneling conductance at reference point $T_{K} /\left\langle T_{K}\right\rangle=1$. $C_{b}$ is also a constant. Now, the theoretical analogue of $G_{0}$ yields $G_{0}=C \rho_{s}^{0}\left(1+C_{b}\right)$.

To compare the values of our theoretical slope $B$ of $T_{K} /\left\langle T_{K}\right\rangle$ vs. $\rho_{s} / \rho_{s}^{0}$, with the experimental slope $B_{e} \simeq$ 2.54 of $T_{K} /\left\langle T_{K}\right\rangle$ vs. $G / G_{0}$ obtained from the data in Fig. 4e, we must take into account that

$$
\frac{G}{G_{0}}=\frac{\rho_{s} / \rho_{s}^{0}+C_{b}}{1+C_{b}} .
$$

It can be readily shown that $B_{e}=\left(1+C_{b}\right) B$. The fact that $\rho_{s} \geq 0$ for the minimum $G$ observed, $G_{\mathrm{m} i n} / G_{0}=0.8$ (see Fig. 4), implies that $C_{b} /\left(1+C_{b}\right)<0.8$, which leads to the upper bound $\sim 4$ for $C_{b}$. The corresponding theoretical value of $B=0.503$ for the NCA method is obtained for $R=0.27$ (Table \). Previously, a lower bound 0.1 was estimated for $\mathrm{Co}$ on $\mathrm{Cu}(111)$ based on the quantum mirage effect assuming $C_{b}=133$. For a more realistic value of the minimum $\rho_{s}$ about $60 \%$ of $\rho_{s}^{0}$ (the value for a surface without scattering sources), we obtain
$B=1.269$ and $R=1$ (Table $\llbracket$ ), i.e., the same coupling of the impurity to the surface states as to the bulk ones.

\section{CONCLUSIONS}

By combining STS, atomic lateral manipulation, and applying a suitable Anderson Hamiltonian for the system, we have demonstrated that surface states have a relevant contribution in the formation of the Kondo state of $\mathrm{Co} / \mathrm{Ag}(111)$. This result can be extended to other noble metal surfaces and provides an important clue in the understanding of more complex correlated electron systems. The sensitivity of $T_{K}$ to the surface state suggests the possibility to tune the coupling strength between a magnetic impurity and its foremost environment using confining nanostructures with size comparable to $\lambda_{F} 28$. In the case of $\mathrm{Co} / \mathrm{Ag}(111)$ we provide a lower bound for the coupling of surface states to Co $3 \mathrm{~d}$-states that is 27 $\%$ of the one to bulk states. Furthermore, we show that a two-channel SU(4) Anderson model (considering both spin and orbital quantum numbers) is more appropriate to describe the Kondo effect than the one-channel SU(2) model. We also show that the proximity of the the surface density of states onset to the Fermi level plays a crucial role in the observed approximately linear dependence of the Kondo temperature with the surface density of states.

\section{Acknowledgements}

We thank N. Lorente and R. Robles for fruitful discussions. We acknowledge financial support provided by the Spanish MINECO (grants MAT2013-46593-C63-P and MAT2016-78293-C6-6-R), as well as the Argentinian CONICET (PIP 112-201101-00832) and ANPCyT (PICT 2013-1045). M.M.L., D.S. and M.R.I. acknowledge the use of SAI-Universidad de Zaragoza.
* Electronic address: aligia@cab.cnea.gov.ar

$\dagger$ Electronic address: serrate@unizar.es

1 J. Kondo, Progress of Theoretical Physics 32, 37 (1964).

${ }^{2}$ V. Madhavan, W. Chen, T. Jamneala, M. F. Crommie, and N. S. Wingreen, Phys. Rev. B 64, 165412 (2001).

3 N. Knorr, M. A. Schneider, L. Diekhöner, P. Wahl, and K. Kern, Phys. Rev. Lett. 88, 096804 (2002).

${ }^{4}$ L. Kouwenhoven and L. Glazman, Physics World 14, 33 (2001).

5 J. Henzl and K. Morgenstern, Phys. Rev. Lett. 98, 266601 (2007).

${ }^{6}$ K. Franke, G. Schulze, and J. Pascual, Science 332, 940 (2011).

7 A. Spinelli, M. Gerrits, R. Toskovic, B. Bryant, M. Ternes, and A. Otte, Nature Commun. 4, 10046 (2015).

8 M. C. Martínez-Velarte, B. Kretz, M. Moro-Lagares, M. H.
Aguirre, T. M. Riedemann, T. A. Lograsso, L. Morellon, M. R. Ibarra, A. Garcia-Lekue, and D. Serrate, Nano Letters (2017).

9 L. Cornils, A. Kamlapure, L. Zhou, S. Pradhan, A. Khajetoorians, J. Fransson, J. Wiebe, and R. Wiesendanger, Physical review letters 119, 197002 (2017).

10 J. Kondo, Phys. Rev. 169, 437 (1968).

11 A. C. Hewson, The Kondo Problem to Heavy Fermions (1997), ISBN 9780521599474.

12 K. Andres, J. E. Graebner, and H. R. Ott, Phys. Rev. Lett. 35, 1779 (1975), URL https://link.aps.org/doi/10.1103/PhysRevLett.35.1779

13 G. Aeppli and Z. Fisk, Comments Condens. Matter Phys. 16, 1192 (1992).

14 N. Roch, S. Florens, V. Bouchiat, W. Wernsdorfer, and F. Balestro, Nature 453, 633 (2008). 
15 J. J. Parks, A. R. Champagne, T. A. Costi, W. W. Shum, A. N. Pasupathy, E. Neuscamman, S. FloresTorres, P. S. Cornaglia, A. A. Aligia, C. A. Balseiro, et al., Science 328, 1370 (2010), URL http://www.sciencemag.org/content/328/5984/1370.abstract

16 S. Florens, A. Freyn, N. Roch, W. Wernsdorfer, F. Balestro, P. Roura-Bas, and A. Aligia, Journal of Physics: Condensed Matter 23, 243202 (2011).

17 R. Vincent, S. Klyatskaya, M. Ruben, W. Wernsdorfer, and F. Balestro, Nature 488, 357 (2012).

18 J. Li, W.-D. Schneider, R. Berndt, and B. Delley, Physical Review Letters 80, 2893 (1998).

19 V. Madhavan, W. Chen, T. Jamneala, M. F. Crommie, and N. S. Wingreen, Science 280, 567 (1998), URL http://www . sciencemag.org/content/280/5363/567.abstract

20 H. C. Manoharan, C. P. Lutz, and D. M. Eigler, Nature 403, 512 (2000).

21 L. Limot, E. Pehlke, J. Kröger, and R. Berndt, Phys. Rev. Lett. 94, 036805 (2005), URL http://link.aps.org/doi/10.1103/PhysRevLett.94.036805

22 D. Serrate, M. Moro-Lagares, M. Piantek, J. I. Pascual, and M. R. Ibarra, The Journal of Physical Chemistry C 118, 5827 (2014).

23 A. Zhao, Q. Li, L. Chen, H. Xiang, W. Wang, S. Pan, B. Wang, X. Xiao, J. Yang, J. G. Hou, et al., Science 309, $1542(2005)$.

${ }^{24}$ T. Komeda, H. Isshiki, J. Liu, Y.-F. Zhang, N. Lorente, K. Katoh, B. K. Breedlove, and M. Yamashita, Nature communications 2, 217 (2011).

25 E. Minamitani, N. Tsukahara, D. Matsunaka, Y. Kim, N. Takagi, and M. Kawai, Physical review letters 109, 086602 (2012).

${ }^{26}$ V. Iancu, K. Schouteden, Z. Li, and C. Van Haesendonck, Chemical Communications 52, 11359 (2016).

27 M. Ormaza, P. Abufager, B. Verlhac, N. Bachellier, M.-L. Bocquet, N. Lorente, and L. Limot, Nature communications 8, 1974 (2017).

28 J. Fernández, M. Moro-Lagares, D. Serrate, and A. A. Aligia, Phys. Rev. B 94, 075408 (2016).

29 O. Újsághy, J. Kroha, L. Szunyogh, and A. Zawadowski, Phys. Rev. Lett. 85, 2557 (2000), URL http://link.aps.org/doi/10.1103/PhysRevLett.85.2557

30 M. Plihal and J. W. Gadzuk, Phys. Rev. B 63, $085404 \quad$ (2001), URL http://link.aps.org/doi/10.1103/PhysRevB.63.085404

31 M. A. Schneider, P. Wahl, L. Diekhöner, L. Vitali, G. Wittich, and K. Kern, Japanese Journal of Applied Physics 44, 5328 (2005), URL http://jjap.jsap.jp/link?JJAP/44/5328/

32 J. Merino and O. Gunnarsson, Phys. Rev. Lett. 93, 156601 (2004), URL http://link.aps.org/doi/10.1103/PhysRevLett.93.156601

33 A. A. Aligia and A. M. Lobos, Journal of Physics: Condensed Matter 17, S1095 (2005), URL http://stacks.iop.org/0953-8984/17/i=13/a=005.

34 Q. Li, S. Yamazaki, T. Eguchi, H. Kim, S.-J. Kahng, J. F. Jia, Q. K. Xue, and Y. Hasegawa, Phys. Rev. B 80, 115431 (2009).

35 Q. L. Li, C. Zheng, R. Wang, B. F. Miao, R. X. Cao, L. Sun, D. Wu, Y. Z. Wu, S. C. Li, B. G. Wang, et al., Phys. Rev. B 97, 035417 (2018), URL https://link.aps.org/doi/10.1103/PhysRevB.97.035417

36 C.-Y. Lin, A. H. Castro Neto, and B. A.
Jones, Phys. Rev. B 71, 035417 (2005), URL https://link.aps.org/doi/10.1103/PhysRevB.71.035417

37 M. Ternes, C. P. Lutz, C. F. Hirjibehedin, F. J. Giessibl, and A. J. Heinrich, Science 319, 1066 (2008).

ct. A. A. Aligia, Phys. Rev. B 88, 075128 (2013).

39 In our own experiments as well as those of Ref. 35 the dip in the differential conductance has a considerable shift to the right of the Fermi level. This is consistent with a Kondo effect with total d occupancy $n_{d}$ near 1 for SU(4) symmetry but is not consistent with either $n_{d} \approx 1$ and $\mathrm{SU}(2)$ symmetry or $n_{d} \approx 2$ for the two-channel model. In these two cases, the Kondo peak is practically at the Fermi level in the Kondo limit 67]. In the supplemental material of Ref. 35 the Friedel sume rule 67] is inverted to estimate $0.4<n_{d}<0.66$ based on the simplest onechannel SU(2) Anderson model. This would indicate that the system is in the intermediate valence regime, instead of the Kondo one. The same analysis for the SU(4) case gives $0.8<n_{d}<1.3$ which is fully consistent with our SU(4) model in the Kondo regime.

40 M. Barral, S. Di Napoli, G. Blesio, P. Roura-Bas, A. Camjayi, L. Manuel, and A. Aligia, The Journal of Chemical Physics 146, 092315 (2017).

41 Calculations in a similar model, indicate that the main effect of a finite $U$ is a shift lo lower energies of the Kondo dip and an increase of its width that can be absorbed renormalizing the (unknown) magnitude of both surface and bulk hybriditations by the same factor [70].

42 J. Li, W.-D. Schneider, and R. Berndt, Phys. Rev. B 56, 7656 (1997), URL https://link.aps.org/doi/10.1103/PhysRevB.56.7656

43 N. Bickers, Reviews of modern physics 59, 845 (1987).

44 P. Anderson, Journal of Physics C: Solid State Physics 3, 2436 (1970).

45 R. Žitko, Phys. Rev. B 84, 085142 (2011), URL https://link.aps.org/doi/10.1103/PhysRevB.84.085142

46 L. Vaugier, A. Aligia, and A. Lobos, Physical Review B 76, 165112 (2007).

47 R. Žitko, Phys. Rev. B 84, 195116 (2011), URL http://link.aps.org/doi/10.1103/PhysRevB.84.195116.

48 A. Aligia, P. Roura-Bas, and S. Florens, Physical Review B 92, 035404 (2015).

49 J. Fernández, F. Lisandrini, P. Roura-Bas, C. Gazza, and A. A. Aligia, Phys. Rev. B 97, 045144 (2018), URL https://link.aps.org/doi/10.1103/PhysRevB.97.045144

50 T. Pruschke and N. Grewe, Zeitschrift für Physik B Condensed Matter 74, 439 (1989).

51 D. E. Logan, M. P. Eastwood, and M. A. Tusch, Journal of Physics: Condensed Matter 10, 2673 (1998).

52 J. Könemann, B. Kubala, J. König, and R. J. Haug, Physical Review B 73, 033313 (2006).

53 J. Kroha et al., Acta Phys. Pol. B 29, 3781 (1998).

54 J. Fernández, A. A. Aligia, P. Roura-Bas, and J. A. Andrade, Phys. Rev. B 95, 045143 (2017).

55 N. S. Wingreen and Y. Meir, Physical review B 49, 11040 (1994).

56 F. Reinert, D. Ehm, S. Schmidt, G. Nicolay, S. Hüfner, J. Kroha, O. Trovarelli, and C. Geibel, Physical review letters 87, 106401 (2001).

57 D. Ehm, S. Hüfner, F. Reinert, J. Kroha, P. Wölfle, O. Stockert, C. Geibel, and H. v. Löhneysen, Physical Review B 76, 045117 (2007).

${ }^{58}$ L. Tosi, P. Roura-Bas, and A. Aligia, Journal of Physics: Condensed Matter 27, 335601 (2015). 
59 S. Di Napoli, P. Roura-Bas, A. Weichselbaum, and A. Aligia, Physical Review B 90, 125149 (2014).

60 P. R. Bas and A. Aligia, Physical Review B 80, 035308 (2009).

61 P. Roura-Bas and A. A. Aligia, Journal of Physics: Condensed Matter 22, 025602 (2009).

${ }^{62}$ K. Haule, S. Kirchner, J. Kroha, and P. Wölfle, Physical Review B 64, 155111 (2001).

${ }^{63}$ L. Tosi, P. Roura-Bas, A. M. Llois, and L. O. Manuel, Physical Review B 83, 073301 (2011).

64 M. A. Schneider, L. Vitali, N. Knorr, and K. Kern, Phys. Rev. B 65, 121406 (2002), URL http://link.aps.org/doi/10.1103/PhysRevB.65.121406

65 K. Nagaoka, T. Jamneala, M. Grobis, and M. F.
Crommie, Phys. Rev. Lett. 88, 077205 (2002), URL http://link.aps.org/doi/10.1103/PhysRevLett.88.077205

66 M. Moro-Lagares, Engineering Spin Structures at the Atomic Scale (Prensas de la Universidad de Zaragoza, 2017), ISBN 978-84-16935-83-3.

${ }^{67}$ L. Tosi, P. Roura-Bas, A. Llois, and A. Aligia, Physica B: Condensed Matter 407, 3263 (2012).

68 A. C. Hewson, The Kondo Problem to Heavy Fermions (1993).

69 Y.-F. Yang, Z. Fisk, H.-O. Lee, J. Thompson, and D. Pines, Nature 454, 611 (2008).

70 J. Fernndez, A. A. Aligia, and A. M. Lobos, EPL (Europhysics Letters) 109, 37011 (2015). 